\title{
SYNERGISM OF DEPRESSANTS IN PETROLEUM SYSTEMS
}

\section{A. Kuprin}

UDC 622.271.3:625.753

Systems of the coking gasoil type with residual and distillate cracking residues were investigated by electrophysical methods. Electrical processes, in particular, electrostatic blocking of high-melting components and formation of asphalt-resin complexes on electrically active centers, as well as mechanical factors, are responsible for the synergism and depressant activity. At 25-30 wt. \% concentrations of additives in the mixtures investigated, a depressant effect was revealed for the first time..

Revealing the mechanism of the effect of depressant additives in cooling is of both scientific and practical importance, for example, in the search for petroleum products that solidify at low temperatures.

Based on the results of a study of the electrophysical characteristics (dielectric constant $\varepsilon$, specific conductivity $\sigma$, conductivity activation energy $E_{\sigma}$, and induced conductivity $U_{i}$ ), we will examine some aspects of the mechanism of the synergistic effect of the depressant additive - 1:1 mixtures of cracking residue (CR) from vacuum resid and distillate cracking-residue (DCR) added to coking gasoil - solvent.

The dielectric parameters were recorded with a type R578 alternating current bridge at a frequency of $1000 \mathrm{~Hz}$, and the electrical resistance was recorded with a E6-13 thermometer. The specific conductivity $(1 / \mathrm{W} \cdot \mathrm{m})$ of the mixtures was calculated with the equation:

$$
\sigma=K / R
$$

where $K$ is the measuring cell constant that takes into consideration its geometric dimensions, $K=5.7691 / \mathrm{m} ; R$ is the resistance of the mixture of petroleum products, W.

The conductivity activation energy was determined with the graphic dependences of $\sigma$ on $1 / T$ with the equation:

$$
E_{\sigma}=4.00116 \cdot 10^{-4} \tan \alpha
$$

Chelyabinsk State University. Translated from Khimiya i Tekhnologiya Topliv i Masel, No. 4, pp. 42 - 44, July - August, 2006. 
where tan a is the tangent of the slope of the linear segment of the curve of $\sigma$ as a function of $1 / T$.

Measurement of the induced potential $U_{\mathrm{i}}$ on a specially created setup consisted of recording (with a U5-6 electrometric amplifier) the difference in potentials between the electrodes in a cylindrical cell in cooling the mixture. The method of measuring $U_{\mathrm{i}}$ is exposed in more detail in [1].

The efficiency of depressant additives is correlated with their polarity, decrease in solvation of the molecules of the high-melting components by the solvent molecules, perturbation of the aggregate stability of the wax dispersion, increase in the compactness of the crystalline aggregates, and formation of associated complexes of the additive molecules and solid hydrocarbons [2,3]. Based on the results of the electrokinetic studies of the effect of depressants on the behavior of suspensions of solid hydrocarbons, hypotheses were advanced concerning the possibility of the electrostatic nature of their effect [4].

In evaluating the depressant properties of resins and asphaltenes (RA), it is necessary to begin not as much with the surface activity on the phase boundary as the ability of the depressants to be adsorbed on crystals of the high-melting components. However, the experimental data that confirm the adsorption mechanism of action of depressant additives are presented in several publications $[5,6]$.

The data on the synergism of cracking residues of residual and distillate origin are basically explained by the effect of the hydrocarbons in the solvent and distillate residue on the viscosity-temperature properties of the mixtures [7]. The previously advanced hypothesis concerning the responsibility of the electrical processes that take place in formation and growth of the crystalline phase for potentiation of depressant activity in the given systems was examined based on the results of the study $[8,9]$.

The presence of polar components - RA - in the mixture causes the formation of dipoles capable of adsorbing these components on the surface of the basic substance. In the investigated mixtures, resins, whose content in CR and DCR are 23 and 16 wt. \%, for example, are polar components. Resins can be incorporated in the crystal lattice of wax or adsorbed on its surface, impeding further crystallization and crystal growth [2, 10].

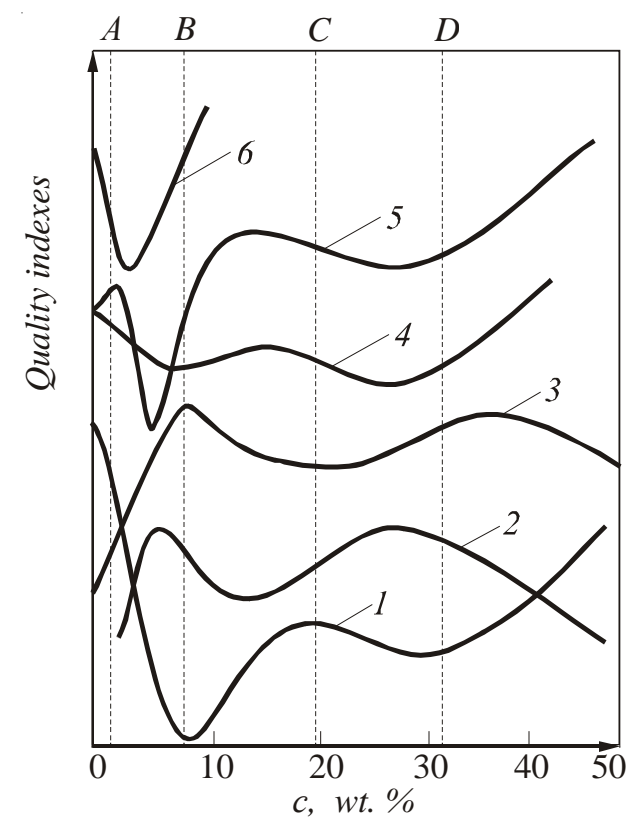

Fig. 1. Quality indexes of coking gasoil mixture vs. cracking residue content $c: 1$ ) solid point (GOST 20278-72); 2) specific conductivity $\sigma ; 3$ ) maximum induced potential $U_{\mathrm{i}}$; 4) conductivity activation energy $\left.E_{\sigma} ; 5\right)$ dielectric constant $\left.\varepsilon ; 6\right)$ surface tension. 
These processes are accompanied by anomalies of the electrophysical properties of these mixtures of petroleum products of a different type (Fig. 1). All electrophysical parameters have two extremal values corresponding to small (5-7 wt. \%) and large (25-30 wt. \%) regions of additive concentrations.

In these regions, $\varepsilon$ and $E_{\sigma}$ decrease, while s and $U_{\mathrm{i}}$ increase. The anomalous change in the electrophysical parameters is most likely due to the formation of charged particles, which becomes possible when a substance is inhomogeneous and consists of several dielectrics with a different dielectric constant [11]. In the substances studied, such conditions are: depressants and waxes differ by almost two times in the dielectric constants (Fig. 2).

The charge $q$ (coulomb) for the individual components of the mixture was calculated in a wide range of temperatures with the experimentally determined values of the capacitance and corresponding potentials using the following equation:

$$
\boldsymbol{q}=\boldsymbol{C} \boldsymbol{U}_{i}
$$

where $C$ is the capacitance, $\mathrm{F} ; U_{\mathrm{i}}$ is the potential, $\mathrm{V}$.

The curves of the charge as a function of the temperature shown in Fig. 3 show that CR and DCR additives acquire electrical charges of opposite signs when the temperature decreases, while the signs of the charges of wax and DCR crystals coincide. These processes basically take place on the boundary of the liquid and solid phases $[12,13]$.

Waxes of normal and isostructure primarily crystallize out of complex petroleum mixtures on cooling. Their content in coking gasoils reaches $50 \mathrm{wt}$. \%. Depressants should be adsorbed on the most active sections of the hydrophobic surface of high-melting hydrocarbon crystals.

The corners of growing $n$-paraffin crystals, which have a dendritic shape with strongly developed sharp facets and solid needle-shaped waxes are such sections [2]. An important electric charge and consequently also electrostatic force that acts on the surrounding charged particles in the high-voltage field created around the sharp facets of wax crystals can be concentrated at such places due to the large radius of curvature.

Nuclei of a solid extrinsic phase which can can energetically separate from solution and form into separate associates or microcrystals (with the opposite electric charge with respect to the high-melting components of the mixture - CR microcrystals), basically adsorb on the sharp facets of the wax crystals.

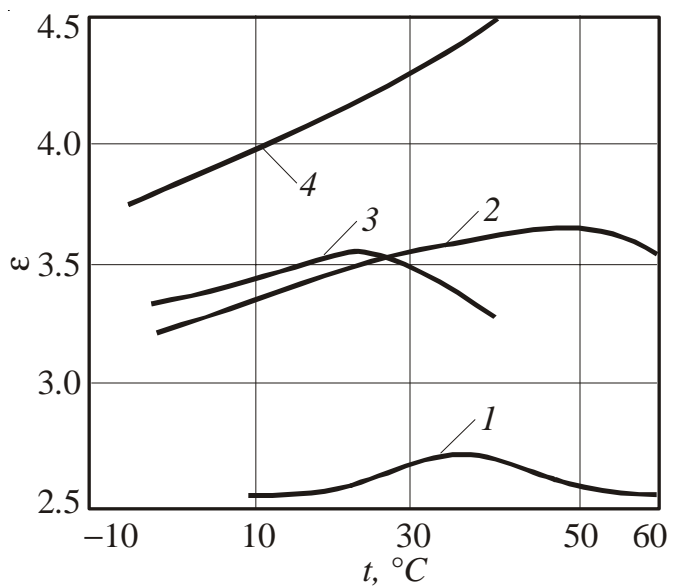

Fig. 2. Dielectric constant $\varepsilon$ vs. temperature $t$ : 1$)$ wax; 2) CR; 3$)$ DCR + CR (1:1); 4) DCR. 
This is first due to the different signs of the charged particles and second, to the electrostatic field forces of these crystals. The predominant adsorption of microcrystals (and individual molecular complexes) of CR on the surface of wax crystals is also confirmed by their lower dielectric constant than for DCR.

At low concentrations of the additives, primarily CR are separated from the mixture due to the lower solubility in coking gasoils in comparison to DCR. Adsorption of molecular complexes of CR on sharp facets (as the most electrically active centers) of growing crystals of high-melting solvent hydrocarbons causes blocking of the surface of the crystals and a sharp reduction in their size.

The difference in the signs of the electric charges of CR and waxes, the uniform distribution of CR and wax complexes over the volume of the mixture, the increase in the mobility and concentration of surface-active centers in the field of the electric charge of the waxes, and the lower dielectric constant of CR than in DCR intensify diffuse processes and adsorption of almost all of the additive.

As a result, most of the quasiliquid phase is poor in the high-melting additive, the viscosity decreases, and solidification of the mixture is shifted to the low-temperature region (see Fig. 1, segment $A B$ ). This is accompanied by an increase in $\mathrm{s}$ and $U_{\mathrm{i}}$ due to an increase in the number of reaction contacts and the number or mobility of electricity carriers, as well as a decrease in $\varepsilon$ due to limitation of the degree of freedom of the individual bonds of the adsorbed molecules. These processes take place at low activation energies, which is also confirmed experimentally.

The insignificant (2-5 wt. \%) amounts of depressants in these mixtures is totally sufficient to inhibit (or retard) ring-shaped growth of wax crystals with the basic electric charge concentrated on the sharp facets, as noted above. This charge is the main cause of deposition of CR and the appearance of conditions for blocking access of wax molecules from the melt to their sharp facets and consequently the maximum synergistic effect at low concentrations of depressants.

A further increase in the concentration of RA in the mixture causes separation of primarily the second component of the depressant - DCR - and the formation of stable extrinsic binary CR $\leftrightarrow$ DCR complexes basically due to their different electric charges. This process extends to a certain concentration interval. It continues until almost all of the CR microcrystals separated and not adsorbed from solution on wax crystals block DCR.

The creation of associated complexes of the colloid type predominates over other factors here, accompanied by an increase in the viscosity, surface tension, and consequently shifting of the solid point of the mixtures toward high values and a change in the electrophysical properties on segment $B C$ (see Fig. 1), the opposite of the change on segment $A B$.

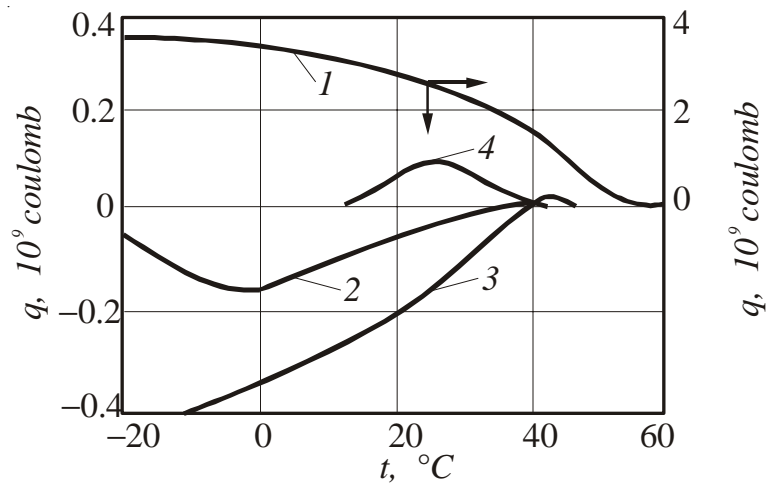

Fig. 3. Charge $q$ vs. temperature $t$ : 1) wax; 2) CR; 3) CR + DCR (1:1); 4) DCR. 
Further adsorption of $\mathrm{CR}$ on the entire surface of the growing crystals takes place with an increase in the concentration of extrinsic additives to 20-30 wt. \%. A change in the orientation of the dipoles of the individual molecules [2] and adsorption of CR microcrystals can alter the sign of the surface charge of the growing wax crystals. Competition between DCR and CR for a place in the surface layer can cause their surface excesses to have different signs. It is preferable to adsorb additives with a smaller dielectric constant.

The solubility of the DCR increases with an increase in the concentration of additives in the mixture. Its molecules and individual microcrystals with the opposite charge with respect to the wax crystals (due to a change in the sign as a result of adsorption of $\mathrm{CR}$ ) diffuse to the surface, forming a second molecular layer and blocking the entire surface of the wax crystals. As a result, the liquid phase is again impoverished, but in DCR, and the mixture again becomes more mobile and a moment arises corresponding to a second synergistic effect (see Fig. 1, segment $C D$ ).

In the 25-30 wt. \% concentration range, the anomalies in the electrophysical and other properties are also repeated. However, the degree is less pronounced than in the first synergistic effect. One reason is the weakness of the intermolecular forces due to the "looseness" of the secondary layers, since the degree of freedom of the individual bonds is less limited, which also causes, for example, a much smaller decrease in the dielectric constant.

The mobility of the charge carriers is also slightly impeded, confirmed by the decrease in the conductivity and induced potential in comparison to their values in the first synergistic effect $[8,9]$.

A further increase in the concentration of additives (over $30 \mathrm{wt}$. \%) primarily causes formation of associates and aggregates in the entire volume, accompanied by structuring of the system and a sharp increase in the solid point of the mixtures (see Fig. 1, segment $D E$ ).

The electric processes responsible for the synergism and depressant activity are accompanied at the minimum by two competing processes: electrostatic blocking of growth of the solid phase of high-melting components and formation of RA complexes by electrically active centers. The driving force of adsorption of depressants on the surface of the growing crystals are electric forces in addition to dispersion forces.

The study of the electrophysical properties of the components of petroleum systems has the goal of obtaining blends with previously defined low-temperature properties.

\section{REFERENCES}

1. V. A. Kuprin and V. I. Tambovtsev, Zavod. Lab., 44, No. 12, 1498-1501 (1978).

2. L. P. Kazakova and S. E. Krein, Physicochemical Principles of Production of Petroleum Oils [in Russian], Khimiya, Moscow (1978).

3. Z. I. Syunyaev, Physicochemical Mechanics of Crude Oils and Principles of Intensifying Refining Processes [in Russian], MINKh i GP im. I. M. Gubkina, Moscow (1979).

4. S. G. Agaev, Candidate Dissertation, I. M. Gubkin Institute of the Petrochemical and Gas Industries, Moscow (1972).

5. V. G. Gutsalyuk and E. A. Yatsenko, Izv. Akad. Nauk KazSSR, Ser. Khim. Nauk, No. 1, 91-94 (1958).

6. V. G. Gutsalyuk, S. R. Rafikov, and Kh. I. Epel'baum, Ibid., No. 2, 26-28 (1963).

7. A. P. Ziknov'ev and P. L. Ol'kov, in: Proceedings of the III Republic Conference "Problem of Exhaustive Refining of Medium- and High-Sulfur Crude Oil Residues [in Russian], UNI, Ufa (1981), pp. 90-92.

8. V. A. Kuprin, in: Safe Behavior in Working in Pits [in Russian], UkrNIIproekt, Kiev (1983), pp. 39-45.

9. V. A. Kuprin, in: Proceedings of the Scientific and Technical Seminar "Theory and Practice of Rational Use of Fuel and Lubricant Materials in Engineering, Chelyabinsk, May 16-20, 1983 [in Russian], Chelyabupropoligrafizdat, Chelyabinsk (1983), pp. 21-22. 
10. A. A. Chesnokov and L. G. Zherdeva, Khim. Tekhnol. Topl. Masel, No. 7, 23-30 (1963).

11. Sh. B. Nad', Dielectrometry [in Russian], Energiya, Moscow (1976).

12. A. M. Mel'nikova, Kristallografiya, 14, No. 3, 548-563 (1969).

13. USSR Inventor's Certificate No. 879420. 